\title{
Aluminum-Activated Citrate and Malate Transporters from the MATE and ALMT Families Function Independently to Confer Arabidopsis Aluminum Tolerance
}

\author{
Jiping Liu ${ }^{1}$, Jurandir V Magalhaes ${ }^{2}$, Jon Shaff ${ }^{1}$ and Leon V Kochian ${ }^{1 *}$ \\ 1. U. S. Plant Soil and Nutrition Laboratory, USDA-ARS, Cornell University, Ithaca, NY 14853, USA. \\ 2. Embrapa Maize and Sorghum, Rod. MG 424, Km 65, 35701-970, Sete Lagoas - MG, Brazil. \\ * Corresponding author: Leon. Kochian@ ars. usda. gov
}

\begin{abstract}
Aluminum ( $\mathrm{Al}$ ) activated root malate and citrate exudation play an important role in $\mathrm{Al}$ tolerance in many plant species. AtALMT1, an Al-activated malate transporter, is a major contributor to Arabidopsis $\mathrm{Al}$ tolerance. Here, we demonstrate that AtMATE encodes an Arabidopsis Al-activated citrate transporter. AtMATE is expressed primarily in roots and is induced by Al. A loss-of-function AtMATE mutant line lacks Al-activated root citrate exudation. An AtALMT1 AtMATE double mutant lacks both Al-activated root malate and citrate exudation and exhibits greater Al sensitivity than the single AtALMT1 mutant. Therefore, AtMATE makes a significant although smaller contribution to Arabidopsis Al tolerance.
\end{abstract}

\section{AtMATE shares the highest sequence identity with SBMATE}

In Arabidopsis, the MATE family contains at least 56 members, which can be further classified into several clusters based on sequence similarity ( $\mathrm{Li}$ et al. , 2002; Rogers and Guerinot, 2002). AtMATE is the Arabidopsis MATE family member with the highest sequence similarity to SbMATE, the recently cloned sorghum Al tolerance gene (Magalhaes et al. , 2007) (Figure 1). The AtFRD3 clade contains 4 members, including AtFRD3 (At3g08040), AtMATE (At1 g51340), At2g38330 and At4g38380.

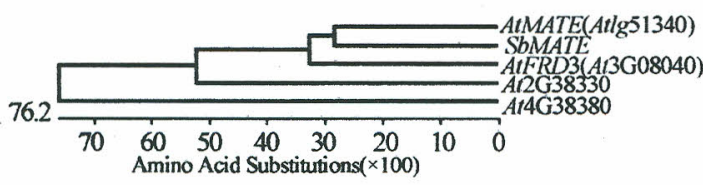

Figure 1 AtMATE shares the highest sequence similarity with SBMATE in Arabidopsis

\section{AtMATE expression in roots is induced by $\mathrm{Al}$ treatment}

By conducting semi-quantitative reverse-transcriptase ( RT )-PCR analysis, we demonstrated that within the AtFRD3 clade, AtMATE is the only member whose gene expression is induced by $\mathrm{Al}$ and is localized primarily to the root, which is the site where $\mathrm{Al}$ tolerance must occur (Figure 2).

\section{AtMATE is responsible for $\mathrm{Al}$-activated root citrate exudation}

In Arabidopsis, $\mathrm{Al}$ induces a high level of malate and lower level of root citrate exudation (WT, Figure $3 \mathrm{a}, \mathrm{b}$ ). The AtALMTI knock-out line (AtALMTI-KO) and the AtMATE-KO line lack $\mathrm{Al}$-activated root malate and citrate exudation, respectively. The AtALMT1 AtMATE double knock-out ( double-KO) line lacks both Al-activated root malate and root citrate exudation.

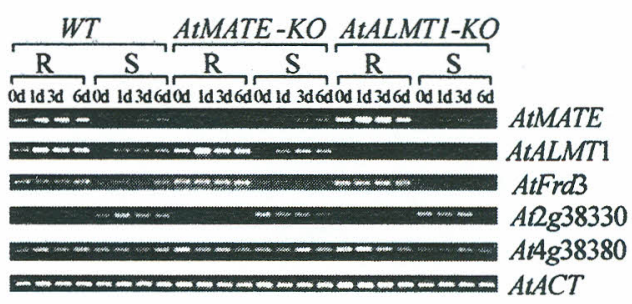

Figure 2 Spatial and temporal gene expression patterns for AtMATE and other members of the AtFRD3 clade in the Arabidopsis MATE family, as well as the major Al tolerance gene, AtALMT1. The 6-day-old Arabidopsis seedlings, wild type (WT), AtMATE knock-out (AtMATE-KO) and AtALMTI knock-out (AtALMT1-KO) lines, were treated with $\mathrm{Al}^{3+}$ for 0 day ( $0 \mathrm{~d}$, the control) , 1 day (1d), 3 days ( $3 \mathrm{~d}$ ), and 6 days (6d). Total RNA was extracted from roots $(R)$ and shoots (S).

The AtMATE AtALMT1 double mutant is highly hypersensitive to $\mathrm{Al}$ stress

Although AtALMT1 is clearly the major determinant of Al tolerance in Arabidopsis as knocking out this gene caused 
an approximately $60 \%$ reduction in $\mathrm{Al}$ tolerance with respect to the wild type at $1.5 \mu \mathrm{M} \mathrm{Al}^{3+}$ activity (Hoekenga et al. , 2006), AtMATE also contribute a moderate (approx. 30\%) component to the overall Arabidopsis Al tolerance. The double AtMATE AtALMTI T-DNA knock-out (Double-KO) line displayed more $\mathrm{Al}$ sensitivity compared to the AtALMTI single mutant (Figure 4).
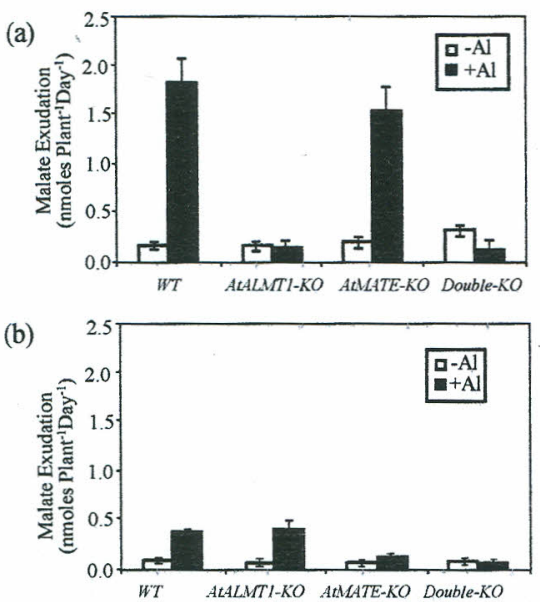

Figure 3 Root malate (a) and citrate (b) exudation under $+/$ - Al conditions for the wild-type (WT) control, the AtMATE knock-out (AtMATE-KO) line, the AtALMT1 knock-out (AtALMTl-KO) line, and the AtMATE, AtALMT1 double knock-out (Double-KO) line

\section{DISCUSSION}

It appears that AtALMT1-mediated Al-activated malate exudation and AtMATE-mediated Al-activated citrate exudation evolved and function independently in conferring the full range of Arabidopsis Al tolerance.

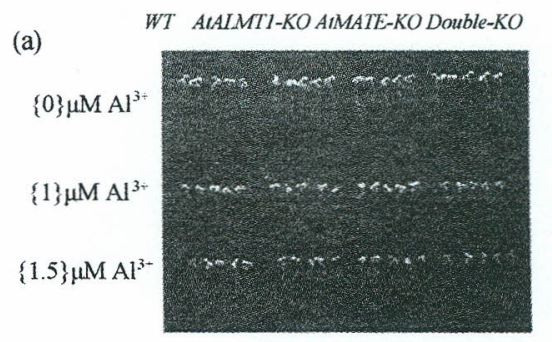

(b)

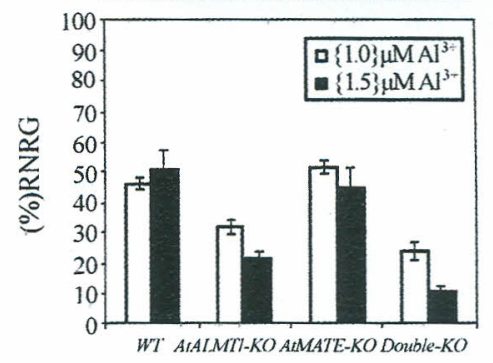

Figure 4 The AtMATE, AtALMT1 double mutant is hypersensitive to $\mathrm{Al}$ stress. (a) Aluminum ( $\mathrm{Al}$ ) tolerance (root growth in nutrition solutions containing $\{0\} \mu \mathrm{M},\{1.0\}$ $\mu \mathrm{M}$, or $\{1.5\} \mu \mathrm{M} \mathrm{Al}^{3+}$ ) for the wild-type (WT) control, the AtALMT1-KO, the AtMATE-KO and the double-KO. ( b ) Aluminum tolerance as measured by percentage of relative net root growth (\% RNRG = root growth in $\mathrm{Al} /$ control root growth $\times 100$ ) in wild type and the three knock-out lines

\section{REFERENCE}

Hoekenga OA, Maron LG, Piñeros MA, et al. 2006. Proc Nat Acad Sci USA, 103: $9738-9743$.

Li L, He Z, Pandey GK, et al. 2002. J Biol Chem, 277 : $5360-5368$.

Magalhaes JM, Liu J, Guimares CT et al. 2007. Nature Genet, 39: $1156-1161$.

Rogers EE, Guerinot ML. 2002. Plant Cell, 14: 1787 $-1791$. 